\title{
Inadequate Sampling Rates Can Undermine the Reliability of Ecological Interaction Estimation
}

\author{
Brenno Cabella ${ }^{1}\left[\right.$, Fernando Meloni ${ }^{2}$ (D) and Alexandre S. Martinez $2,3, *(\mathbb{D})$ \\ 1 Instituto de Física Teórica, Universidade Estadual Paulista (UNESP), Rua Dr. Bento Teobaldo \\ Ferraz 271, 01140-070 São Paulo, Brazil; cabellab@gmail.com \\ 2 Faculdade de Filosofia, Ciências e Letras de Ribeirão Preto (FFCLRP), Universidade de São Paulo (USP), \\ Avenida Bandeirantes 3900, 14040-901 Ribeirão Preto, São Paulo, Brazil; fernandomeloni@usp.br \\ 3 Instituto Nacional de Ciência e Technologia de Sistemas Complexos (INCT-SC), Rua Dr. Xavier Sigaud 150, \\ Urca, 22290-180 Rio de Janeiro, Brazil \\ * Correspondence: asmartinez@usp.br; Tel.: +55-16-3315-3720
}

Received: 28 March 2019; Accepted: 29 April 2019; Published: 30 April 2019

check for updates

\begin{abstract}
Cycles in population dynamics are abundant in nature and are understood as emerging from the interaction among coupled species. When sampling is conducted at a slow rate compared to the population cycle period (aliasing effect), one is prone to misinterpretations. However, aliasing has been poorly addressed in coupled population dynamics. To illustrate the aliasing effect, the Lotka-Volterra model oscillatory regime is numerically sampled, creating prey-predator cycles. We show that inadequate sampling rates may produce inversions in the cause-effect relationship among other artifacts. More generally, slow acquisition rates may distort data interpretation and produce deceptive patterns and eventually leading to misinterpretations, as predators becoming preys. Experiments in coupled population dynamics should be designed that address the eventual aliasing effect.
\end{abstract}

Keywords: temporal aliasing effect; ecological methods; sampling rates; cyclic dynamics; predator-prey system; population biology

\section{Introduction}

Quantitative sampling provides the most important information source for ecological modeling. An important example, but not yet fully understood, is the periodic species abundance cycles in population dynamics. These cycles may appear in coupled systems, in which two or more elements (species or climate) interact in a cause-effect relationship. In Bulmer [1], lags between species cycles (phase shifts) were used to infer the relationship between different species in Canada.

Also, the historic data series observed by trappers working for Hudson's Bay Company, MacLulich [2] and Elton and Nicholson [3] found regular cycles in the population of Snowshoe Hares (Lepus americanus) and Canadian Lynx (Lynx canadensis). Their abundance have been matched, and showed an overlap with a small delay. The system was interpreted from the perspective of trophic interactions, as a regular predator-prey system, which was first labeled the Lotka-Volterra model (LVM) [4]. Some years later, the model became more robust, considering finite limits in the oscillatory predation rate [5]. Although predator-prey models are intuitively coherent and produce qualitative patterns found in nature, such models provide poor adjustment to field data, so their empiricism is still controversial [6].

There is a scientific consensus that better samples in field experiments lead to better interpretation of the real pattern. Effects caused by inappropriate sampling have already been addressed in the context of spatial influence on population dynamics or by the numerical insufficiency of samples $[7,8]$. 
However, period between samples are generally neglected and species interaction are especially prone to data misinterpretation when inappropriate sampling rates are used. The most common problem associated with slow sample rates is the aliasing effect. The usual concern of this artifact is the misidentification of a signal frequency [9-11]. However, aliasing may also occur in multivariate signals and its effect goes beyond frequency changes. In a bivariate coupled system the lag between prey and predators (phase shift) may also be compromised.

A thorough search in the scientific literature shows that the aliasing effect is poorly explored in Ecology, and its consideration may have deep implications. For instance, delays in coupled systems are ordinarily interpreted as competition effects in Ref. [12]. Also, Benicà and collaborators $[13,14]$ have studied a long time series of plankton communities, applying regular samples to measure several species. The authors have found that the cause-effect relationship suggests a chaotic food web.

This manuscript is organized as follows: Firstly, we present the temporal aliasing effect and the Lotka-Volterra model. Next, we numerically solve the model and sample it with different rates. Finally, we present the results and artifacts due to poor sampling i.e., apparent inversion of cause-effect relationship, increased cycle period and synchronism.

\section{Aliasing the Lotka-Volterra Model}

Temporal aliasing effect occurs when the sampling rate is not fast enough compared to the system natural cycle period. For example, in movies, the spiked wheels on horse-drawn wagons sometimes appear to turn backwards, the "wagon-wheel effect", which is depicted in Figure 1. A wheel indeed turns clockwise, but due to the slow sampling by the camera (number of frames per second), a filmed wheel appears to turn counter-clockwise. This effect can be avoided considering the Nyquist-Shannon sampling theorem [15], which states that given a time series with minimum period $\tau$, the equally spaced intervals between samples $T_{s}$ must be smaller than half the minimum period, i.e., $T_{s}<\tau / 2$.

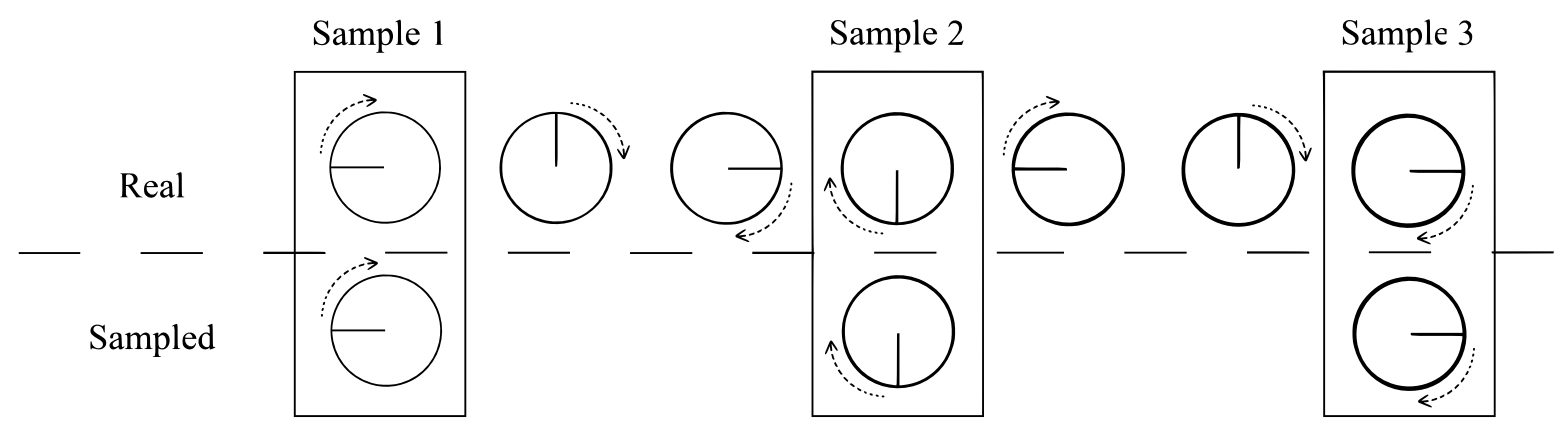

Figure 1. Aliasing wheel. Example of an aliasing effect in the clockwise rotation of a wheel. The visualized behavior on film is a counter-clockwise rotation, known as the wagon-wheel effect. The long time interval between samples explains this curiosity.

In Ecology, cycles are extensively found in systems in which species interact with each other and with the environment [6]. To illustrate how the aliasing effect may mislead the interpretation of population abundance cycles, consider a simple prey-predator interaction described by the LVM: $d x / d t=x(\alpha-\beta y)$ and $d y / d t=y(\delta x-\gamma)$, where $x(t)$ and $y(t)$ are the prey and predator population densities at time $t$, respectively, $\alpha$ is the prey growth rate in the absence of predators, $\gamma$ is the predator death rate in the absence of prey, and $\beta$ and $\delta$ are related to the interaction strength between both species. The LVM equations have two fixed points: the mutual extinction, $E_{1}\left(x^{*}, y^{*}\right)=(0,0)$, and the neutral center, $E_{2}\left(x^{*}, y^{*}\right)=(\gamma / \delta, \alpha / \beta)$. Solutions around the singular point $E_{2}$ are cycles with period $\tau=2 \pi / \sqrt{\alpha \gamma}$. Although the LVM is not adequate to quantitatively describe real-world community dynamics, here it is suitable because of its cause-effect relation: the number of predators increases (decreases) after the prey abundance increases (decreases). 
To demonstrate how sampling rates can change the patterns in predator-prey systems, we have numerically obtained the cyclic dynamic pattern using the LVM. The Lotka-Volterra differential equations have been implemented in MatLab ${ }^{\circledR}$ language, and their solutions have been obtained using the Dormand-Prince method [16]. Dormand-Prince is currently the default method in the ode45 solver for MatLab ${ }^{\circledR}$. The standard LV dynamics was obtained with the following parameters: $\left(x_{0}, y_{0}\right)=(1.01,0.99), \alpha=\beta=\delta=\gamma=2 \pi$ and $t \in[0,1000]$ with resolution $10^{-3}$. The model parameters have been set to produce a unitary oscillation period $\tau=1$ near the equilibrium point $E_{2}$.

Figure 2a shows the prey (dashed line) and predator (full line) population cycles. Next, the prey (empty circle) and predators (filled circle) were sampled within fixed time intervals, $T_{s}$. We repeated the procedure, increasing $T_{S}$ from $\tau / 10$ until $1.1 \tau$. For each sampling rate, we interpolated the points to build the respective time series to infer the original series. Based on the peaks of the time series, we inferred the oscillation period and the dephasing of predator and prey abundances. In all the cases, we considered all the individuals from both populations and sampling does not alter species interactions nor population densities. Moreover, any spacial effect is considered negligle. In this way, individuals are considered to be homogeneously spatially distributed (random mixing hypothesis), their number is large enough therefore we may neglect deviations around their mean densities, leading to the isolation of the sampling rate effect.

\section{Sampling Effects}

For $T_{S}<\tau / 2$, the system real cycle period is correctly retrieved as expected by the Nyquist-Shannon theorem as displayed in Figure $2 b$, with $T_{s}=\tau / 10$. As $T_{S}$ increases, the signal becomes increasingly biased. Figure $2 c, d$ depict different patterns even though the sample period is the same $T_{S}=0.4$. In Figure $2 \mathrm{e}, T_{s}=0.48$, interleaved synchrony and anti-synchrony occurs for the same series. Even though the Nyquist-Shannon criteria is satisfied, i.e. the oscillation period of both species can be properly retrieved, the phase relation between them is disrupted. For exact $T_{S}=0.5$, limiting value for the Nyquist-Shannon criteria, two possible behaviors emerge: In Figure $2 \mathrm{f}$ prey and predators abundances are anti-correlated; or perfectly correlated as shown Figure $2 \mathrm{~g}$. Similarly to the cases presented in Figure $2 c, d$, the observed series will depend on the initial sample, i.e., the phase of the cycle where the first sample is obtained. A further increase in $T_{S}$ causes an inversion of the prey-predator cycle and also an enhancement of the population cycle period is observed. In Figure $2 \mathrm{~h}$, $T_{S}=0.9$, an increasing (decreasing) in prey population is followed by a decrease (increase) in predators, which is the opposite expected from a prey-predator relationship.

The inverted cycle oscillations persist for even greater values of $T_{S}$ as the oscillation period increases to $T_{s} \rightarrow \tau$. When $T_{s}=\tau$, there are no oscillations, as depicted in Figure 2i. For even greater values for $T_{s}$, the original dynamics is retrieved but with extended cycle period, shown in Figure $2 \mathrm{j}$, $T_{s}=1.1$. The patterns presented from Figure $2 \mathrm{~b}-\mathrm{i}$ repeat for $k \tau<T_{s}<(2 k+1) \tau$ where $k=0,1,2, \ldots$.

The effect of cycle inversion and frequency change is summarized in Figure 3. When prey and predator series are acquired with adequate sample rate, prey and predator abundances and lag between them are properly retrieved, see Figure $3 \mathrm{a}$. Figure $3 \mathrm{~b}$ presents the phase portrait in prey vs. predator plot where the cycle turns counterclockwise. However, when the sample rate is not adequate, an inversion of the cycle occurs, as shown in Figure $3 \mathrm{c}$ and seen in the phase portrait of Figure $3 \mathrm{~d}$. Figure 3e shows the changes in the observed frequency and prey-predator cycle direction as function of sample period $T_{S}$. Note that as $T_{S}$ increases, the original direction of the cycle may be retrieved (counterclockwise) but the observed period $\left(\tau_{0}\right)$ will be always longer than the original. 

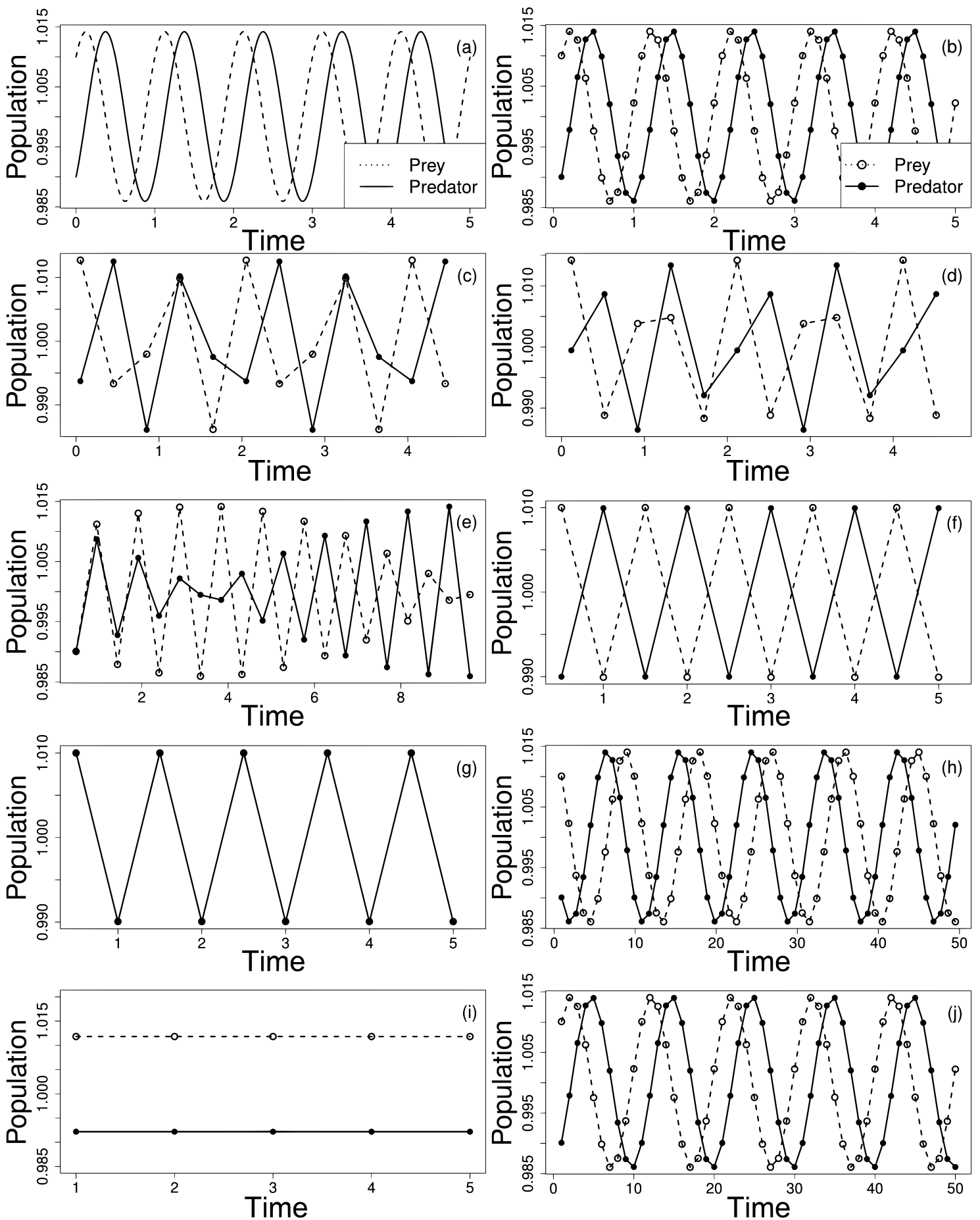

Figure 2. (a) Prey (dashed line) and predator (full line) population cycles obtained with the Lotka-Volterra model (LVM), with oscillation period $\tau=1$. The LVM dynamics can generate different patterns due only to sampling rate effects. In the above panels, prey and predator abundances are represented by an empty and full circles, respectively. (b) $T_{S}=0.1$, the time series correctly retrieve the LVM behavior. (c) $T_{s}=0.4$, peaks seems to synchronize every two cycles. (d) $T_{s}=0.4$, same sample period as in (c) but with different pattern. (e) $T_{S}=0.48$, synchronous and anti-synchronous patterns are present in the same series. With $T_{S}=0.5$ (Nyquist limit), two possible behaviors appears: (f) anti-synchronous cycles or (g) fully synchronized. (h) $T_{S}=0.9$, an inversion and an extension of cycle period may be interpreted as preys eating predators. (i) $T_{S}=1$ no oscillations are observed. (j) $T_{s}=1.1$, the original dynamics is retrieved but with extended cycle period. 

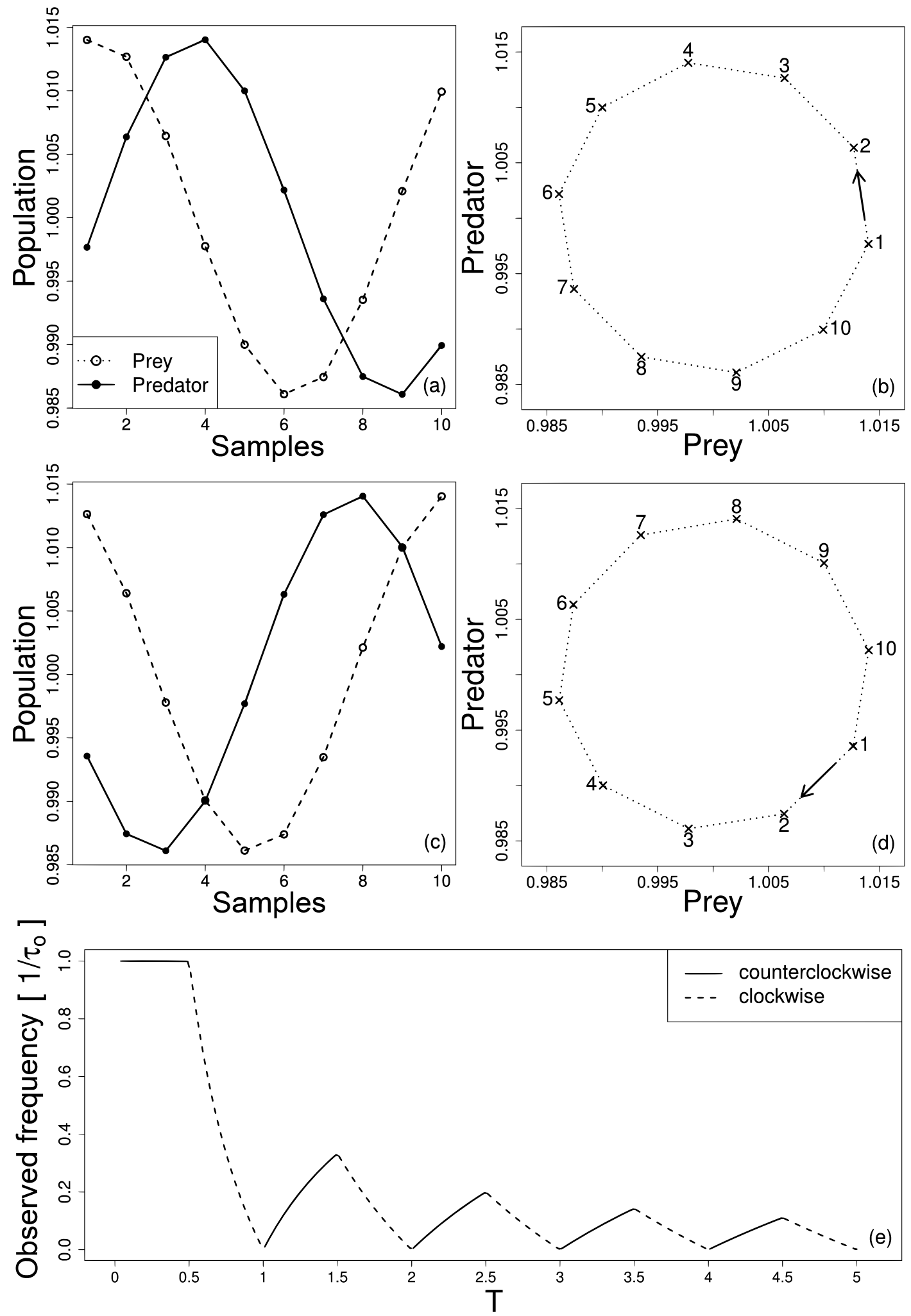

Figure 3. (a) Prey and predator series with adequate sample rate $\left(T_{S}=0.1\right)$ and corresponding phase portrait in (b). In a prey vs. predator plot, the cycle turns counterclockwise. (c) Prey and predator series with inadequate sample rate $\left(T_{S}=0.9\right)$ and corresponding phase portrait in $(\mathbf{d})$. In this case the cycle turns clockwise. (e) changes in the observed frequency and prey-predator cycle direction as function of sample period $T_{s}$. Each sample in (a) and (c) is represented in (b) and (d) respectively. 
A very simple and controlled oscillatory behavior, such the one LVM simulates, may produce different patterns in time series due only to inappropriate sampling rates, as shown in Figure $2 \mathrm{~b}-\mathrm{g}$. Therefore, ecological interactions may be misinterpreted if data were collected with insufficiently sampling rates. In real world systems, this difficulty is amplified because the populations' periodicity is not necessarily constant and/or many species interactions may tangle the dynamics even more.

Aliasing should also be better evaluated in many other circumstances, such as the coupled aerosol-cloud-rain system, because the LVM is applied to modeling [17]. The influence of climate anomalies has been investigated as a driver of periods in population dynamics, as in the hare-lynx system $[18,19]$. Species abundance rates are the basis for evaluation of biological control success in crops, and in such cases, aliasing may have great financial consequences [20]. Sampling effects also have implications for biological conservation and species management, as in marine ecosystems, where population levels are used as a criterion to regulate fishing [21]. Further, some theoretical approaches about the trade-offs in Ecology and Evolution also concern predator-prey systems, trophic interactions or population cycles interactions [22-25]. Aliasing effect should also be considered on decision-making of public policies regarding national parks, fish stocks and hunting schedule, since the prediction of population levels often relies on sampled data.

\section{Conclusion}

We have stressed the importance of the aliasing effect in retrieving the behavior of ecological interactions. We have numerically demonstrated that slow sampling rates may lead to data misinterpretation. Aliasing is an often neglected effect that should be carefully considered when real data is used to model systems interaction. The aliasing hypothesis may provide new insights into old problems in Ecology and Biology. This result also highlights the importance of the field researchers, that can provide with realistic estimates for the population cycle periods, avoiding any circumstantial sampling with poor experimental designs.

Author Contributions: A.S.M., B.C. and F.M. designed the research; B.C. and F.M. performed the research and wrote computational codes; A.S.M. verified numerical results; B.C. and F.M. wrote the paper; A.S.M., B.C. and F.M. edited the paper. All authors reviewed the manuscript.

Funding: A.S.M. holds grants from CNPq 309851/2018-1, B.C. thanks Capes for support through a PNPD fellowship (88882.317469/2019-01) and F.M. acknowledges grant FAPESP 2013/06196-4.

Acknowledgments: We would like to thank the organizers of the Summer Course on Mathematical Methods in Population Biology, Roberto André Kraenkel and Paulo Inácio de Knegt López de Prado, where the hare-lynx paradox was first presented to us.

Conflicts of Interest: The authors declare no conflict of interest.

\section{Abbreviations}

The following abbreviation is used in this manuscript:

LVM Lotka Voltera model

\section{References}

1. Bulmer, M.G. A Statistical Analysis of the 10-Year Cycle in Canada. J. Anim. Ecol. 1974, 43, 701-718. [CrossRef]

2. Barbosa, P.; Caldas, A.; Riechert, S.A. Species Abundance Distribution and Predator-Prey Interactions: Theoretical and Applied Consequences. In Ecology of Predator-Prey Interactions; Oxford University Press: Oxford, UK, 2005; pp. 344-368.

3. Elton, C.S.; Nicholson, M. The ten year cycle in numbers of lynx in Canada. J. Anim. Ecol. 1942, 11, $215-244$. [CrossRef]

4. Odum, E.P. Fundamentals of Ecology; Saunders: Philadelphia, PA, USA, 1953.

5. Rosenzweig, M.L.; MacArthur, R.H. Graphical representation and stability conditions of predator-prey interactions. Am. Nat. 1963, 97, 209-223. [CrossRef] 
6. Murray, J.D. Mathematical Biology: I. An Introduction; Springer: New York, NY, USA, 2012.

7. Kery, M.; Dorazio, R.M.; Soldaat, L.; van Strien, A.; Zuiderwijk, A.; Royle, J.A. Trend estimation in populations with imperfect detection. J. Appl. Ecol. 2009, 46, 1163-1172. [CrossRef]

8. Kishida, O.; Trussell, G.C.; Mougi, A.; Nishimura, K. Evolutionary ecology of inducible morphological plasticity in predator-prey interaction: Toward the practical links with population ecology. Popul. Ecol. 2010, 52, 37-46. [CrossRef]

9. Oppenheim, A.V.; Schafer, R.W. Sampling of continuous-time signals. In Discrete-Time Signal Processing; Prentice Hall: Upper Saddle River, NJ, USA, 1999; pp. 140-150.

10. Green, D.G. Time Series and Postglacial Forest Ecology. Quat. Res. 1981, 15, 265-277. [CrossRef]

11. Ford, D.E.; Thornton, K.W. Time and length scales for the one-dimensional assumption and its relation to ecological models. Water Resour. Res. 1979, 15, 113-120. [CrossRef]

12. Vandermeer, J. Coupled oscillations in food webs: Balancing competition and mutualism in simple ecological models. Am. Nat. 2004, 163, 857-867. [CrossRef] [PubMed]

13. Benincà, E.; Huisman, J.; Heerkloss, R.; Jöhnk, K.D.; Branco, P.; van Nes, E.H.; Scheffer, M.; Ellner, S.P. Chaos in a long-term experiment with a plankton community. Nature 2008, 451, 822-825. [CrossRef] [PubMed]

14. Benincà, E.; Jöhnk, K.D.; Heerkloss, R.; Huisman, J. Coupled predator-prey oscillations in a chaotic food web. Ecol. Lett. 2009, 12, 1367-1378. [CrossRef] [PubMed]

15. Nyquist, H. Certain Topics in Telegraph Transmission Theory. Trans. Am. Inst. Electr. Eng. 1928, 47, 617-644. [CrossRef]

16. Dormand, J.R.; Prince, P.J. A family of embedded Runge-Kutta formulae. J. Comput. Appl. Math. 1980, 6, 19-26. [CrossRef]

17. Koren, I.; Feingold, G. Aerosol-cloud-precipitation system as a predator-prey problem. Proc. Natl. Acad. Sci. USA 2011, 108, 12227-12232. [CrossRef] [PubMed]

18. Yan, C.; Stenseth, N.C.; Krebs, C.J.; Zhang, Z. Linking climate change to population cycles of hares and lynx. Glob. Chang. Biol. 2013, 19, 3263-3271. [CrossRef]

19. Stenseth, N.C. Canadian hare-lynx dynamics and climate variation: Need for further interdisciplinary work on the interface between ecology and climate. Clim. Res. 2007, 34, 91-92. [CrossRef]

20. Snyder, W.E.; Chang, G.C.; Prasad, R.P. Conservation Biological Control: Biodiveristy Influences the Effectiveness of Predators. In Ecology of Predator-Prey Interactions; Oxford University Press: Oxford, UK, 2005; p. 324.

21. Hunsicker, M.E.; Ciannelli, L.; Bailey, K.M.; Buckel, J.A.; Wilson White, J.; Link, J.S.; Essington, T.E.; Gaichas, S.; Anderson, T.W.; Brodeur, R.D.; et al. Functional responses and scaling in predator-prey interactions of marine fishes: Contemporary issues and emerging concepts. Ecol. Lett. 2011, 14, 1288-1299. [CrossRef] [PubMed]

22. Weitz, J.S.; Levin, S.A. Size and scaling of predator-prey dynamics. Ecol. Lett. 2006, 9, 548-557. [CrossRef]

23. Cortez, M.H. Comparing the qualitatively different effects rapidly evolving and rapidly induced defences on predator-prey Interactions. Ecol. Lett. 2011, 14, 202-209. [CrossRef] [PubMed]

24. Kalinkat, G.; Schneider, F.D.; Digel, C.; Guill, C.; Rall, C.B.; Brose, U. Body masses, functional responses and predator-prey stability. Ecol. Lett. 2013, 16, 1126-1134. [CrossRef] [PubMed]

25. Schneider, F.D.; Scheu, S.; Brose, U. Body mass constraints on feeding rates determine the consequences of predator loss. Ecol. Lett. 2012, 15, 436-443. [CrossRef] [PubMed]

(C) 2019 by the authors. Licensee MDPI, Basel, Switzerland. This article is an open access article distributed under the terms and conditions of the Creative Commons Attribution (CC BY) license (http://creativecommons.org/licenses/by/4.0/). 